\title{
ACOSO LABORAL, AUTODESPIDO Y TUTELA LABORAL: UN CASO DE ESTUDIO
}

\author{
Andrés Dighero Eberhard* \\ Universidad de Chile
}

\begin{abstract}
RESUMEN: El Acoso Laboral es un proceso sistemático de socavación y destrucción de la persona que recién ahora nuestro legislador ha reconocido como un mal presente en las relaciones laborales y que atenta, precisamente, contra la dignidad de la persona en la relación laboral. No obstante lo violento de este fenómeno, el legislador no había establecido medios eficaces para tutelar la dignidad de la persona sino hasta la promulgación de la ley 20.087. Tampoco tuvo claridad sobre cómo abordarlo como fenómeno ni los derechos que se veían afectados, evidencia de lo anterior es la torpe conceptualización inserta en el artículo 2 del Código del Trabajo. Este artículo analiza como este fenómeno se relaciona con las herramientas que el ordenamiento jurídico le entrega a la persona para combatirlo, en especial, el instituto del autodespido y el procedimiento de tutela laboral, desde un punto de vista tanto ontológico como teleológico.
\end{abstract}

Palabras clave: Acoso Laboral, autodespido, derechos fundamentales, procedimiento de tutela laboral

\begin{abstract}
The Workplace Bullying is a systematic process of undermining and destruction of the person you just now our legislature has recognized as an evil present in labor relations and attentive, precisely, the dignity of the person in the employment relationship. Violent Notwithstanding this phenomenon, the legislature had not established effective ways to protect the dignity of the person until the enactment of the law 20,087. Nor had clarity on how to approach rights phenomenon and how they are affected, evidence of this is the clumsy conceptualization inserted in Article 2 of the Labor Code. This article discusses how this phenomenon relates to the tools the law gives to the person to fight, especially, the institute's internal stagnation and labor guardianship proceeding, from a standpoint of both ontological and teleological.
\end{abstract}

Keywords: Workplace Harassment or Mobbing, self dismissal, fundamental rights, labor tutelage procedure

\section{INTRODUCCIÓN}

Hasta el año 2012, a falta de una definición legal, el Mobbing o Acoso Moral Laboral había sido conceptualizado por la Doctrina. En este sentido, los primeros en tratarlo en extenso fueron los profesores Gamonal y Prado quienes conceptualizaron el Acoso Moral Laboral como un:

“... proceso conformado por un conjunto de acciones u omisiones, en el ámbito de las relaciones laborales públicas y privadas, en virtud de las cuales uno o más sujetos acosadores crean un ambiente laboral hostil e intimidatorio respecto de uno o más acosados, afectando gravemente su dignidad personal y dañando la salud del o los afectados con miras a lograr distintos fines de tipo persecutorio"1.

En este trabajo utilizaré las siguientes abreviaturas: Código del Trabajo (CT), Real Academia de la Lengua Española (RAE). Gamonal, Sergio; Prado, Pamela. El Mobbing o acoso moral laboral. Santiago, Chile: LexisNexis, 2006, p.22. 
Andrés Dighero Eberhard / Acoso laboral, autodespido y tutela laboral: un caso de estudio

Hoy en día, luego de variados proyectos de ley y de una extenuante discusión, la Ley No 20.607, publicada en el Diario Oficial el día 8 de agosto de 2012, vino a ver la luz llenando aparentemente este vacío conceptual de nuestra legislación laboral, definiendo el Acoso Laboral y tipificándolo como una causal de caducidad del contrato de trabajo que puede ser utilizada tanto por la vía del despido directo como del autodespido. Es así como en su artículo $1^{\circ}$ esta ley dispone:

"Agrégase en el inciso segundo del artículo 2º a continuación del punto aparte (.) que pasa a ser punto seguido (.), la siguiente oración: "Asimismo, es contrario a la dignidad de la persona el acoso laboral, entendiéndose por tal toda conducta que constituya agresión u hostigamiento reiterados, ejercida por el empleador o por uno o más trabajadores, en contra de otro u otros trabajadores, por cualquier medio, y que tenga como resultado para el o los afectados su menoscabo, maltrato o humillación, o bien, que amenace o perjudique su situación laboral o sus oportunidades en el empleo".

Decimos "aparente" porque ya la jurisprudencia entendía bien este fenómeno y, luego de la dictación de la reforma procesal laboral, con el procedimiento de tutela laboral, la conceptualización resultó ser algo más simbólico que efectivo.

En cuanto a su campo de aplicación, la Dirección del Trabajo, en ejercicio de sus facultades propias, dicta el Dictamen No 3519/034, de 9 de agosto de 2012, señalando que:

"Conforme a lo expuesto en párrafos anteriores, es posible inferir que el legislador ha concebido las conductas constitutivas de acoso laboral en términos amplios, en forma tal que permita considerar como tales todas aquellas conductas que impliquen una agresión física hacia el o los trabajadores afectados o que sean contrarias al derecho que les asiste, así como las molestias o burlas insistentes en su contra, además de la incitación a hacer algo, siempre que todas dichas conductas se ejerzan en forma reiterada, cualquiera sea el medio por el cual se someta a los afectados a tales agresiones u hostigamientos.

Ahora bien, la norma en comento exige, además, que tales conductas ocasionen menoscabo, maltrato o humillación al o los trabajadores afectados, debiendo entenderse por tales, según ya se precisara, cualquier acto que cause mengua o descrédito en su honra o fama o que implique tratar mal de palabra u obra o que los hiera en su amor propio o en su dignidad, o bien, que amenacen o perjudiquen la situación laboral o las oportunidades de empleo de dichos afectados.

A lo anterior cabe agregar que, al utilizar la expresión "[...] o bien que amenace o perjudique su situación laboral o sus oportunidades en el empleo", el legislador ha entendido que la conducta de acoso laboral se configura no sólo cuando la acción del empleador o del o los trabajadores ocasiona un perjuicio o daño laboral directo en su situación al interior de la empresa, sino también cuando por la creación de un ambiente hostil y ofensivo del trabajo, se pone en riesgo su situación laboral u oportunidades en el empleo".

Dicho Dictamen, como resultado de una desafortunada técnica interpretativa, fue más lejos y conceptualizó, en base a la propia ley, el Acoso Laboral como:

"todo acto que implique una agresión física por parte del empleador o de uno o más trabajadores, hacia otro u otros dependientes o que sea contraria al derecho que les asiste a estos 
últimos, así como las molestias o burlas insistentes en su contra, además de la incitación a hacer algo, siempre que todas dichas conductas se practiquen en forma reiterada, cualquiera sea el medio por el cual se someta a los afectados a tales agresiones u hostigamientos y siempre que de ello resulte mengua o descrédito en su honra o fama, o atenten contra su dignidad, ocasionen malos tratos de palabra u obra, o bien, se traduzcan en una amenaza o perjuicio de la situación laboral u oportunidades de empleo de dichos afectados".

Decimos desafortunada puesto que, siendo rigurosos, tanto el concepto legal como la interpretación dada por la Dirección del Trabajo adolecen de dos problemas graves. En efecto, atendida dicha pobre técnica normativa utilizada en la conceptualización del Acoso Laboral la Dirección del Trabajo, en base al Diccionario de la RAE, su Dictamen ya citado presenta inicialmente al Acoso Laboral resaltándolo en primer lugar como una "agresión física".

Resta decir que el carácter ontológico del acoso laboral está dado por el ataque, la agresión y el carácter teleológico, por la afección psicológica que genera y en el deterioro de la salud mental de la víctima. En otras palabras, al agregar un adjetivo calificativo como "física" restringe innecesariamente el concepto, al punto de perder de vista que lo importante es la agresión, y su resultado en la persona; no logrando entender que lo medular es la creación de un ambiente hostil que atenta contra la dignidad del trabajador.

Además, y volviendo ahora sobre el problema de la definición que el legislador ha dado, es importante destacar que erróneamente se definió el Acoso Laboral de forma muy similar al Acoso Sexual, y esto es problemático porque la definición que nos entrega la ley o 20.005 hacía dificultoso entender que se recogía el acoso sexual ambiental. Sin perjuicio de que ese problema en el acoso sexual ambiental fue relativamente superado por medio de una interpretación jurisprudencial acertada, volver a caer en ese mismo error no parece comprensible, más todavía cuando precisamente el Acoso Laboral en su esencia se define o se entiende como la creación de un ambiente hostil. Y el problema, cabe la reiteración, reside precisamente en esta idea: la creación de un ambiente hostil no se encuentra en lo absoluto debidamente recogido con este concepto que ha dado la ley No 20.607 al definir el Acoso Laboral. Esto, obviamente, trae consigo el mismo problema interpretativo que existió con el acoso sexual ambiental, pero magnificado considerando que Acoso Laboral se entiende en si como la creación de un ambiente laboral hostil que atenta contra la dignidad de la persona del trabajador.

Resulta asimismo criticable del referido concepto legal, el hecho que se reconozca un ilícito de resultado y no de mera actividad, lo cual importa que el trabajador deba acreditar no solamente las acciones, sino que también el resultado, lo cual sin duda debilita la protección ante el acoso laboral, más aún si se considera que este puede consistir simplemente en la creación de un ambiente laboral hostil que de suyo atenta contra la dignidad de la persona del trabajador.

Por último, se puede también criticar la definición legal ya que identifica al Acoso Moral Laboral como "Acoso Laboral", lo que terminológicamente hablando es incorrecto atendida la relación de género a especie existente entre el Acoso Laboral en sentido amplio y el Acoso Moral Laboral. Con todo, esto no representa un mayor problema, por lo que utilizaremos como términos idénticos "Acoso Laboral" y "Acoso Moral".

Por otra parte, estructuralmente hablando, el acoso moral, de igual manera que el sexual, puede importar una dinámica vertical, sea en su forma descendente (Bossing) o ascendente, y una de tipo horizontal (entre compañeros o pares de trabajo) y sus características fundamentales son: 
Andrés Dighero Eberhard / Acoso laboral, autodespido y tutela laboral: un caso de estudio

a) Se produce con ocasión del desenvolvimiento de relaciones laborales.

b) Es siempre un conjunto de conductas de cierta duración en el tiempo que por acción u omisión producen un ambiente o entorno laboral hostil, que si se miraran de forma aislada parecerían inofensivos o inocuos. Este es uno de los criterios diferenciadores con el Acoso Sexual, que precisamente se puede configurar con una conducta aislada.

c) Produce como efecto el cortar o derribar los canales o redes de comunicación de la víctima.

d) Teleológicamente, tiene una finalidad de naturaleza persecutoria.

En cuanto a su reconocimiento positivo el Acoso Moral Laboral, previo a la publicación de la Ley 20.607, no tenía un tratamiento legislativo sistemático o específico en nuestro ordenamiento jurídico. Sólo de forma indirecta el artículo 145-J aludía al Mobbing al prohibir que se excluyera arbitrariamente al trabajador de artes y espectáculos de los correspondientes ensayos y de las demás actividades preparatorias para el ejercicio de su actividad artística.

No obstante ello, la jurisprudencia, tanto administrativa como judicial, había reconocido el acoso moral como una derivación de la idea que el ordenamiento jurídico laboral, en su función integradora, entrega una protección al trabajador frente a este tipo de actos fundándose esencialmente en el denominado contenido ético-jurídico del contrato de trabajo, lo que desde el punto de vista práctico implica que el empleador culpable de este tipo de acoso incurriría en la causal de caducidad del contrato de trabajo de "incumplimiento grave de las obligaciones que impone el contrato", esgrimible por el trabajador por medio de la institución conocida como autodespido o despido indirecto, accediendo con ello a las indemnizaciones por término de contrato más los recargos que la ley establece para estos casos, como igualmente a la acción de tutela laboral de derechos fundamentales, con el fin de obtener la declaración de la nulidad de los actos vulneratorios y las indemnizaciones correspondientes, incluida; en su oportunidad, la indemnización por daño moral, sin perjuicio de otras vías de protección que eventualmente pudiesen proceder.

Adicionalmente, y no obstante los vacíos legales denunciados, esta jurisprudencia se ha sustentado en algunas normas constitucionales como son el artículo 19 en sus numerales $1^{\circ}, 4^{\circ}$ y $5^{\circ}$, como también en tratados internacionales ratificados por Chile, luego de la modificación constitucional de 1989 que introdujo un inciso $2^{\circ}$ al artículo $5^{\circ}$, que dispone que "el ejercicio de la soberanía reconoce como limitación el respeto a los derechos esenciales que emanan de la naturaleza humana. Es deber de los órganos del Estado respetar y promover tales derechos, garantizados por esta Constitución, así como por los tratados internacionales ratificados por Chile y que se encuentren vigentes".

\section{PROTECCIÓN DEL TRABAJADOR AFECTADO POR ACOSO LABORAL}

Como ya se mencionó, las acciones u omisiones constitutivas de acoso laboral pueden derivar directamente del empleador (Mobbing vertical descendente) o de uno o más trabajadores en contra de otro u otros de sus pares (Mobbing horizontal), pero aún en este caso indudablemente existe responsabilidad del empleador al permitir que tales acciones se produzcan, cuando pudiendo tomar medidas efectivas para impedir estos actos no las ha tomado, atendida el deber general que impone el artículo $184 \mathrm{del}$ CT al empleador de proteger eficazmente la vida de sus trabajadores. 
En presencia de acciones u omisiones constitutivas de acoso laboral nuestro sistema jurídico entrega al afectado las siguientes vías de defensa judicial:

\subsection{Recurso de Protección}

Esta acción, mal llamado "Recurso", procede en atención a que las conductas constitutivas de acoso laboral al ser desplegadas, pueden atentar en contra el derecho fundamental de la vida e integridad física y psíquica de la persona, tutelado por el artículo $19 \mathrm{~N}^{\circ} 1$ inciso primero de nuestra norma fundamental; contra el derecho a la honra y a la vida privada reconocida en el artículo $19 \mathrm{~N}^{\circ}$ 4; contra la inviolabilidad del hogar y de toda forma de comunicación privada resguardada por el artículo 19 No 5, todos ellos relacionados con el artículo 20 de la Constitución Política de la República que asegura el imperio del derecho y la protección del afectado.

No obstante, en los hechos esta vía no resulta ser la más adecuada atendido el carácter extraordinario de esta acción, que además por su naturaleza no se adapta a las cuestiones de orden laboral. A ello cabe agregar que el Recurso de Protección resulta inoperante para la declaración de nulidad que requieren los actos constitutivos de acoso laboral y para el pago de indemnizaciones al afectado, lo que exige un proceso ante un juez de grado ${ }^{2}$.

\subsection{Acción Civil de Indemnización de Perjuicios}

Los actos de acoso laboral constituyen vulneraciones a derechos fundamentales, por lo que existe para el afectado la facultad de accionar por incumplimiento del contrato de trabajo fundado en el hecho de que la legislación laboral lo convierte en garante de la vida e integridad de sus trabajadores al imponerle el deber y obligación legal de tomar todas las medidas necesarias para proteger eficazmente la vida y salud de los trabajadores en virtud de los dispuesto en el artículo 184 inciso $1^{\circ}$ del CT.

Efectivamente, al vulnerarse la dignidad del trabajador se infringen sus derechos fundamentales, de lo que se derivan daños de distinta naturaleza, y el daño según un principio general en responsabilidad civil debe repararse íntegramente, dejando a la víctima del daño en el estado que se encontraría de no haberlo sufrido ${ }^{3}$.

En el caso del Mobbing, al no contemplarse en el Código del Trabajo normas especiales es posible recurrir a las disposiciones del Código Civil siempre que no se opongan a los principios del derecho del trabajo. En tal caso al acoso moral laboral se le aplicará el estatuto de responsabilidad contractual o extracontractual, dependiendo del vínculo entre acosador y acosado, lo que tendrá incidencia en la determinación de la competencia, pues si el acosador es empleador es competente el juez del trabajo y en otros casos lo será el juez civil.

\subsection{DESPIDO DIRECTO E INDIRECTO}

El número 2 del artículo $1^{\circ}$ de la ley No 20.607, de 2012, agregó dentro conductas indebidas de carácter grave, debidamente comprobadas que tipifican las causales de caducidad del artículo 160 No 1 del Código del Trabajo, una letra f) que expresa: “f) Conductas de acoso laboral”, de manera que el empleador puede despedir sin indemnización a sus trabajadores que incurran en

\footnotetext{
2 Véase en UGarte, José Luis. Derechos, trabajo y privacidad. Santiago, Chile: AbeledoPerrot LegalPublishing, 2011, en pie de página 106 , p. 71.

Véase artículos 2315 y 2329 del Código Civil.
} 
Andrés Dighero Eberhard / Acoso laboral, autodespido y tutela laboral: un caso de estudio

esta conducta y, a su turno, de acuerdo a la modificación que esta misma ley introdujo al artículo 171, el mismo trabajador/víctima puede poner término unilateralmente a su contrato de trabajo, instando por el pago de las indemnizaciones asociadas a la terminación y, eventualmente, de las indemnizaciones por daño moral. Pero esta acción solamente puede fundarse en ciertas causales de caducidad del contrato de trabajo, a saber:

a) Acoso laboral vertical: Previo a la dictación de la nueva ley sobre acoso laboral, dentro de las causales que habilitaban a un trabajador para desprenderse de su relación laboral por actos que resultasen imputables a su empleador, relativos al acoso laboral, las causales relevantes, de acuerdo con el artículo 171 del CT, se consideran tipificadas en los números $1^{\circ}$, en sus letras a), b), c), d) y e); $5^{\circ}$ y $7^{\circ}$ del artículo 160 , disposiciones que recogen la falta de probidad, el acoso sexual, las vías de hecho, las injurias proferidas al trabajador y las conductas inmorales, respectivamente, siempre que revistan el carácter de "graves" y "debidamente comprobadas"; las acciones o imprudencias temerarias que afectan la seguridad o la actividad de los trabajadores o la salud de éstos y el incumplimiento grave de las obligaciones que impone el contrato de trabajo.

En ese plano, las conductas que se entendían como acoso laboral, para poder hacer uso de las causales citadas, tenían que revestir alguna de las formas que las mismas enunciaban. Por lo anterior, el proceso de acoso laboral se debía enmarcar bajo cualquiera de las conductas tipificadas por la ley y únicamente era perseguible por medio del despido indirecto, cuando configuraban justamente alguna de las conductas sancionadas.

Con la nueva definición legal el trabajador puede accionar directamente de despido indirecto por acoso laboral, sin tener la necesidad de encuadrar forzadamente las conductas constitutivas de acoso laboral en otra causal, más que la que se encuentra recogida en el $\mathrm{N}^{\circ} 1$, letra f) del artículo 160 del Código del Trabajo, esto es, "conductas de acoso laboral".

b) Acoso laboral horizontal: En este caso nos parece que la causal aplicable será en primer lugar la establecida en el numeral $7^{\circ}$ del artículo 160, esto es, el incumplimiento grave de las obligaciones que le impone el contrato, la que habilita con más fuerza a poner término al contrato de trabajo por parte del afectado por las conductas de acoso, pudiendo también invocarse directamente la causal de la letra f) del artículo $160 \mathrm{~N}^{\circ}$ 1, a la luz del denominado acoso laboral ambiental, es decir, el que el trabajador ha sufrido en su ambiente laboral con el consiguiente menoscabo que ello le ha implicado. Ello, toda vez que, que existiría para este caso una la obligación legal del empleador de mantener un ambiente libre de acoso laboral, a la luz que el mandato del artículo 184 del CT le impone la obligación de tomar todas las medidas necesarias para proteger de manera eficaz la vida y la salud de sus trabajadores.

Claramente, la existencia de conductas de acoso laboral por parte de los pares de un trabajador influye y afecta la salud de éstos en su globalidad, la que debe ser protegida por el empleador en su totalidad, sobre todo cuando es el empleador quien dicta dentro de su empresa el reglamento interno de orden, donde se plasma la obligación de establecer las normas comunes que se deben observar para velar y garantizar un ambiente laboral digno y de mutuo respeto entre los trabajadores. Todo lo anterior, sin dejar de mencionar que el propio artículo 2 del CT establece un deber general para todos los sujetos del contrato del trabajo de fundar las relaciones laborales en un trato compatible con la dignidad. Así lo ha reconocido expresamente el Dictamen antes comentado, ${ }^{4}$ que sobre el particular expresa: 
"En el mismo sentido, cobra particular relevancia en la prevención y represión del acoso laboral, la norma del artículo 184 del citado cuerpo legal, que establece el deber de protección en el ámbito laboral, en los siguientes términos: "El empleador estará obligado a tomar todas las medidas necesarias para proteger eficazmente la vida y salud de los trabajadores, informando de los posibles riesgos y manteniendo la condiciones adecuadas de higiene y seguridad en las faenas, como también los implementos necesarios para prevenir accidentes y enfermedades profesionales".

Finalmente, a este respecto debe tenerse presente lo dispuesto en el inciso segundo del artículo 171 del CT que establece que:

"el trabajador que ponga término a su contrato de trabajo por la causal de acoso laboral, tendrá derecho a reclamar de su empleador, además de las ya indicadas y en forma simultánea, otras indemnizaciones a que pudiere tener derecho". Pero por otra parte, el inciso sexto dispone: "Si el trabajador hubiese invocado la causal de la letra b) o f) del número 1 del artículo 160, falsamente o con el propósito de lesionar la honra de la persona demandada y el tribunal hubiese declarado su demanda carente de motivo plausible, estará obligado a indemnizar los perjuicios que cause al afectado. En el evento que la causal haya sido invocada maliciosamente, además de la indemnización de los perjuicios, quedará sujeto a las otras acciones legales que procedan".

\subsection{Acción de Tutela Laboral por Derechos Fundamentales}

En atención a que los derechos fundamentales que se ven afectados en casos de acoso laboral son la vida, integridad física y psíquica de la persona, la inviolabilidad del hogar y de la correspondencia privada, la prohibición de discriminación y la protección a la vida privada y la honra, estos son objeto de tutela por parte del legislador laboral a través de un procedimiento especial al que puede acceder el trabajador, regulado en los artículos 485 y siguientes del CT.

Como lo ha expresado la Dirección del Trabajo:

"la intención del legislador ha sido, por una parte, sancionar el acoso laboral ejercido en las condiciones previstas en la nueva normativa como contrario a la dignidad de la persona, por tratarse de una conducta ilícita, que lesiona diversos bienes jurídicos que derivan de dicho derecho, tales como la integridad física y psíquica y la igualdad de oportunidades, teniendo, de esta forma, por fundamento, el respeto y promoción de los derechos fundamentales de las personas en el ámbito de su trabajo, resguardados por la Constitución y las leyes, destacándose, entre estas últimas, por ser directamente aplicable en la especie, la del Título III del Libro I del Código del Trabajo, del Reglamento Interno, que en su artículo 153, inciso $2^{\circ}$, prescribe: "Especialmente, se deberán estipular las normas que se deben observar para garantizar un ambiente laboral digno y de mutuo respeto entre los trabajadores"5. 
Andrés Dighero Eberhard / Acoso laboral, autodespido y tutela laboral: un caso de estudio

Este procedimiento se puede iniciar estando vigente la relación laboral, en los términos que se establecen en el artículo 485 y 495 del CT, y en caso que se haya puesto término a la relación laboral produciéndose la vulneración "con ocasión del despido", de acuerdo con el artículo 489 y 495 del mismo texto autoritativo.

Es indudable que este procedimiento de tutela laboral presenta mayores ventajas para la víctima de acoso laboral, que todas las otras acciones previstas en la legislación nacional, en especial, porque:

- $\quad$ Permite que el juez del grado en su primera resolución ponga término, de oficio o a petición de parte, a los efectos nocivos o perjudiciales del acto vulneratorio impugnado que puedan causar efectos irreversibles al trabajador que es víctima del acoso.

- $\quad$ Proporciona al trabajador una herramienta novedosa, esto es, el de la prueba indiciaria que aligera el peso de la prueba, pues cuando de los "antecedentes aportados resulten indicios suficientes de la vulneración alegada, el denunciado quedará en la necesidad jurídica de explicar los fundamentos de las medidas adoptadas y su proporcionalidad" (Art. 493 del CT).

- Obliga al sentenciador a establecer las acciones tendientes a la reparación de las consecuencias derivadas de la vulneración de los derechos fundamentales afectados, junto con determinar las indemnizaciones que hubiese lugar, logrando de tal forma el efecto reparatorio necesario para toda víctima de vulneración de derechos fundamentales.

\section{COMPATIBILIDAD DE LA ACCIÓN DE DESPIDO INDIRECTO CON LA ACCIÓN DE TUTELA LABORAL}

La doctrina laboral ${ }^{6}$ en su mayoría ha estado de acuerdo en que el ejercicio de la acción de tutela laboral es compatible con el autodespido -salvo algunas voces disidentes ${ }^{7}$, pero en cambio:

"la respuesta de la judicatura ha sido vacilante, toda vez que pese a existir una clara tendencia a reconocer la procedencia de la acción de despido indirecto, en la mayoría de los casos reconociendo de su ejercicio vía hipótesis del artículo 489, no ha habido consistencia, si la forma correcta de proposición, en esa hipótesis, era en forma conjunta o en subsidio de la acción de tutela de derechos fundamentales; así encontramos sentencias en que se ha acogido conjuntamente ambas acciones, otras en que se ha rechazado la acción de tutela de derechos fundamentales con ocasión del despido y se ha entrado al conocimiento de la acción subsidiaria de despido indirecto, acogiéndose esta última"8.

Véase en UGarte, José Luis. Tutela de derechos fundamentales del trabajador. 1ª edición. Santiago, Chile: LegalPublishing, 2009, pp. 94 y 95; y él mismo. "Su majestad el formalismo: Comentario sentencia Corte de Apelaciones de Valdivia Rol No 47-2012", en: Revista de Derecho Laboral y Seguridad Social, Vol. I-2013, Santiago, Chile, pp. 149-152.

Para una postura en contrario véase LANATA FuENZALIDA, Gabriela. "El despido indirecto y el nuevo procedimiento de tutela de derechos fundamentales del trabajador”, en: Revista Chilena de Derecho del Trabajo y de las Seguridad Social, Vol. 2, n 3, Santiago, Chile, 2011, pp. 55-77.

8 Mesías Torres, Cesar. "Acción de Tutela de Derechos Fundamentales y de Despido Indirecto". Parte II, en: Revista Laboral Chilena, Enero 2012, p. 85. 
Sin perjuicio de lo anterior, con respecto a los razonamientos a favor del ejercicio de la acción de despido indirecto con ocasión de un despido vulneratorio. Ejemplar es la postura desarrollada por el magistrado Álvaro Flores Monardes quien en resumen expresa que:

"Que una interpretación teleológica y armónica del artículo 171 del Código del Trabajo, permite sostener su compatibilidad con la acción recogida en el artículo 489 del mismo texto ${ }^{9}$, toda vez que:

a) ...tratándose de las causales de caducidad que sirven de causa de pedir al instituto (número 1, 5 y 7 del artículo 160 del Código del Trabajo), imputables a la conducta incumplidora grave y culpable del empleador, la extinción tiene como causa directa y antecedente inmediato la conducta de este.

b) por la decisión del autodespido, está estrecha e indisolublemente ligada a la acción procesal que la ha de seguir, desde que la conclusión de los servicios por decisión del trabajador sólo tiene sentido si seguidamente se ejercen las acciones indemnizatorias correspondientes, asociadas a la causal de caducidad imputable al empleador, las que no se comprenden en un derecho tutelar, disminuidas respecto de aquellas a que tienen derecho los dependientes exonerados por decisión directa del empleador.

c) Porque no es posible -sin infringir el mandato de no discriminación- razonar atribuyéndose el instituto del autodespido, desde la pura literalidad del precepto, un alcance limitado, diverso a los que origina el despido patronal. Llevado el razonamiento al terreno de la acción de tutela de derechos fundamentales y la norma contenida en el artículo 489, en lo pertinente, se llegaría al absurdo de que un trabajador que ve vulnerado sus derechos fundamentales, queda en situación desmedrada, privado de la acción de amparo, por el solo hecho de hacer aquello que la doctrina y la jurisprudencia han predicado por años de manera uniforme, cuando sostienen ilícitos de tal entidad hacen insostenible la relación de trabajo y provocan un quiebre irreversible del vínculo, el que no puede proseguir y le exigen poner término de inmediato al mismo, en un camino, en que el pie orzado para accionar, lo pone el empleador al incumplir el contrato.

d)...y porque -finalmente- planteada una duda exegética como la que se formula, aún en el escenario procesal, el principio protector impone al intérprete optar por aquella más favorable al trabajador y más protectora de sus derechos, ellos en relación con el principio Pro Cives o Pro Homine de interpretación constitucional (artículo 29, literal d) de la Convención Americana de Derecho Humanos)"10.

Lamentablemente, a pesar de los poderosos argumentos a favor, poco a poco, con un criterio formalista y solapadamente economicista, la Corte Suprema hizo un llamado al "orden" a las 
Andrés Dighero Eberhard / Acoso laboral, autodespido y tutela laboral: un caso de estudio

Cortes de Apelaciones y Jueces del Grado, secundada por las Cortes de Valdivia ${ }^{11}$, Valparaíso ${ }^{12}$ y Arica $^{13}$, al pronunciarse en contra de la compatibilidad de dichas acciones.

Esencialmente, del fallo recaído sobre recurso de unificación de la Corte Suprema ${ }^{14}$, dicha Corte ha impuesto la siguiente Doctrina:

“I. De acuerdo a los artículo 485, 486 y 489 del Código del Trabajo, el procedimiento de tutela laboral está destinado a dar protección efectiva de los derechos fundamentales del trabajador y puede impetrarse cuando: a) la vulneración de garantías se produce durante la vigencia de la relación laboral y b) cuando la vulneración se produce al término de la misma, con ocasión del despido del trabajador, adquiriendo en este caso, la desvinculación el carácter de atentatoria contra los derechos fundamentales. Esta segunda hipótesis se trata de aquella situación en que el empleador toma la decisión de desvincular al trabajador, con vulneración de los derechos fundamentales protegidos.

Siendo claro el sentido del artículo 489 inciso $1^{\circ}$, no corresponde desatender su tenor literal, en cuanto preceptúa nítidamente que la procedencia de esta acción de tutela, ha sido regulada para el evento específico en que la vulneración de garantías constitucionales se produzca con ocasión del despido de un trabajador o, en otras palabras, cuando es el empleador el que proceda a despedir al trabajador en las condiciones anotadas (considerandos $4^{\circ}$ y $5^{\circ}$ de la sentencia que acoge el recurso de nulidad).

Esta conclusión se ve reforzada cuando la norma en comento confiere la acción exclusivamente al "trabajador afectado", debiendo entenderse que ha sido afectado por un despido atentarlo de derecho fundamentales. En consecuencia, en la medida que el artículo 489 del Código del Trabajo regula una situación especial y particular de vulneración de derechos, que opera con ocasión del despido que lleva a cabo el empleador y que considera una indemnización sancionatoria, tal texto debe ser objeto de una interpretación restrictiva, esto es, que se ajuste a la especificidad de la norma descartando su aplicación a situaciones no previstas en ella, como ocurría con la consideración del autodespido que se funda en vulneración de derechos fundamentales. En suma, fundada la demanda de tutela laboral en el despido indirecto de la demandante que habría tenido lugar por conductas del empleador demandado, vulnerando sus derechos fundamentales protegidos por la acción prevista en el artículo 489 inciso $1^{\circ}$ del Código del Trabajo, ella debe ser rechazada por improcedente, toda vez que la referida acción se contempla exclusivamente para el caso de un despido atentarlo de tales derechos cuando es el empleador quien ha decidido desvincular a su dependiente (considerandos $6^{\circ}$ y $8^{\circ}$ de la sentencia que acoge el recurso de nulidad y $3^{\circ}$ de la sentencia de reemplazo)".

\footnotetext{
Véase sentencia pronunciada por la Corte de Apelaciones de Valdivia recaída en recurso de nulidad, Rol N 47-2012.

Véase sentencias pronunciadas por la Corte de Apelaciones de Valparaíso recaídas en recurso de nulidad, Roles N ${ }^{\circ}$ 6074-2010, 6995-2010 y 4196-2010.

13 Véase sentencia pronunciada por la Corte de Apelaciones de Arica recaída en recurso de nulidad, Rol No 15-2011.

14 Sentencia recaída en recurso de unificación, Rol N².202-2012, de 18/01/2013.
} 
Por otra parte, habría resultado más interesante y difícil de rebatir que el Tribunal Supremo hubiese utilizado el argumento que -vía acción del artículo 485 del Código del Trabajo- se hubiese solicitado una indemnización adicional por concepto de daño moral, a modo de reparar el daño experimentado por aquel trabajador víctima de conductas de acoso laboral. En este sentido, tal vez el caso más emblemático de esta vía puede ser encontrado en el caso de la "Trabajadora musulmana”, que siendo objeto de atentados contra su derecho a la vida, integridad psíquica y física y a la no discriminación por su condición religiosa, en un contexto de acoso laboral, solicitase a modo de reparación una indemnización por daño moral.

A pesar de haberse dado la razón a la demandante y haber fallado a su favor ${ }^{15}$, queda claro que esta vía presenta una serie de inconvenientes que en clave de derechos fundamentales es imposible de obviar. En primer lugar, la interposición de esta acción haría aún más insostenible la situación de aquel trabajador que siendo víctima de acoso laboral como fue este caso, pudiese resistir los embates de una empleador más molesto y cegado por la 'ira' de aquel que osa acusarlo con el dedo de su conducta inmoral, proponga arremeter con más fuerza a "deshacerse" de aquel trabajador, que cuando inicio las conductas de hostigamiento laboral. En segundo lugar, un escenario como el descrito tornaría no solo insostenible la relación laboral, sino que profundizaría los efectos nocivos y menoscabadores de la dignidad de la persona ${ }^{16}$, consecuencia o efecto que basalmente el legislador ha querido prevenir y reparar con la consagración de este procedimiento, porque es poco creíble que conminado por el Tribunal, en su primera resolución -también poco común de ver -, decida cesar en su actuar.

Es más, a pesar que esta acción pueda ser más atractiva respecto del ejercicio de facultades del empleador esencialmente inocuas pero que afectan garantías fundamentales en su implementación -generalmente menos intensas porque no singularizan necesariamente un trabajador en particular-; es igualmente cierto que aquellas situaciones donde al ser dirigidas y con un trasfondo espurio son extremadamente intensas, como son los casos de hostigamiento laboral, a todas luces se aprecia como un procedimiento inoportuno y poco práctico, que en vez de detener el comportamiento atentatorio razonablemente podrían incluso hasta incentivar un aumento en su intensidad.

\section{COMENTARIOS FINALES}

La reciente promulgación de la ley 20.607 vino a develar una problemática que el legislador no había previsto, pero que ya tenía una solución hasta hace poco adecuada en la jurisprudencia.

Desafortunadamente, los palos de ciego de la ley vienen a enturbiar las aguas al conceptualizar el Mobbing laboral de una forma en que nada aporta al panorama actual; mucho menos hace la interpretación de la Dirección del Trabajo.

\footnotetext{
15 El Primer Juzgado de Letras del Trabajo de Santiago condenó a la Empresa a pagar \$5.000.000.- por indemnización por daño moral, más reajuste e intereses, y una multa 60 UTM por haber vulnerado el Código del Trabajo. Véase sentencia RIT T-38-2010, caratulada "INSPECCION PROVINCIAL DEL TRABAJO DE SANTIAGO con TP CHILE S.A.", dictada por el Primer Juzgado de Letras del Trabajo de Santiago.

16 Recordemos que existe mandato expreso del legislador consagrado en el inciso segundo del artículo 2 del Código del Trabajo, en sentido a velar por que las relaciones laborales deban "siempre fundarse en un trato compatible con la dignidad de la persona"; mandato que con mayor razón el sentenciador procurar sea observado en todo momento durante la vigencia de la relación laboral, cuando se han producido cuestiones que han sido llevadas a su conocimiento y resolución.
} 
Andrés Dighero Eberhard / Acoso laboral, autodespido y tutela laboral: un caso de estudio

En cuanto a la forma de interactuar de la acción de tutela laboral con el autodespido o despido indirecto, podemos mencionar que no vemos incompatibilidad alguna entre estas normas y la tutela laboral, toda vez que el artículo 489 del Código del Trabajo, al hablar de una de vulneración que se produce "con ocasión del despido", no establece que sea un requisito el que tenga que ser directamente el empleador quien tenga que ponerle término a la relación laboral, sino que la vulneración se produzca justamente a propósito de la terminación y como su causa.

Es más, no compartimos en lo absoluto lo señalado por algunas Cortes de Apelaciones y por la Corte Suprema respecto de la necesidad de una interpretación restrictiva de las normas, toda vez que en clave de derechos fundamentales los formalismos como estos hacen perder todo sentido a la tutela laboral de dichos derechos. La normas sobre interpretación tienen un rango inferior a los mismos bienes cautelados y por tanto no puede tomarse a la ligera una conducta que vulnera dichos bienes puesto que la solución, la tutela laboral, interpretada de manera restrictiva, no puede ser llevada a cabo, con el consecuente sinsentido de mantener en la total impunidad aquél que se aprovecha de su propio dolo.

De hecho, una interpretación restrictiva llevaría al absurdo que el mismo empleador se pueda aprovechar de su propio dolo al no despedir al trabajador, buscando evitar la sanción del artículo 489 ya citado, forzándolo a renunciar, cerrando por dentro la puerta del Mobbing Laboral.

\section{BIBLIOGRAFÍA}

\section{$\underline{\text { Libros y artículos }}$}

Gamonal, Sergio; Prado, Pamela. El Mobbing o acoso moral laboral. Santiago, Chile: LexisNexis, 2006.

Lanata Fuenzalida, Gabriela. "El despido indirecto y el nuevo procedimiento de tutela de derechos fundamentales del trabajador", en: Revista Chilena de Derecho del Trabajo y de las Seguridad Social, Vol. 2, n 3, Santiago, Chile, 2011, pp. 55-77.

Mesías Torres, Cesar. "Acción de Tutela de Derechos Fundamentales y de Despido Indirecto”. Parte II, en: Revista Laboral Chilena, Enero 2012.

Ugarte, José Luis. Derechos, trabajo y privacidad. Santiago, Chile: AbeledoPerrot LegalPublishing, 2011.

Ugarte, José Luis. Tutela de derechos fundamentales del trabajador. 1a edición. Santiago, Chile: LegalPublishing, 2009.

Ugarte, José Luis. "Su majestad el formalismo: Comentario sentencia Corte de Apelaciones de Valdivia Rol N $47-$ 2012”, en: Revista de Derecho Laboral y Seguridad Social, Vol. I-2013, Santiago, Chile.

Documento de la Dirección del Trabajo

Dirección del Trabajo. Ord. No 3519/034, de 9 de agosto de 2012. En http://www.dt.gob.cl/legislacion/1611/w3article-63379.html

Sentencias

Corte Suprema, recurso de unificación de jurisprudencia, 18 de enero de 2013, Rol № 2.202-2012.

Corte de Apelaciones de Valdivia, recurso de nulidad, 07 de mayo de 2012, Rol № 47-2012.

Corte de Apelaciones de Valparaíso, recurso de nulidad, Rol N 6074-2010.

Corte de Apelaciones de Valparaíso, recurso de nulidad, Rol № 6995-2010.

Corte de Apelaciones de Valparaíso, recurso de nulidad, Rol № 4196-2010.

Corte de Apelaciones de Arica, recurso de nulidad, Rol N 15-2011.

Juzgado de Letras del Trabajo de Santiago, RIT T-11-2009.

Juzgado de Letras del Trabajo de Santiago, RIT T-38-2010. 